\title{
Three-dimensional geometrical changes of the human tibialis anterior muscle and its central aponeurosis measured with three-dimensional ultrasound during isometric contractions
}

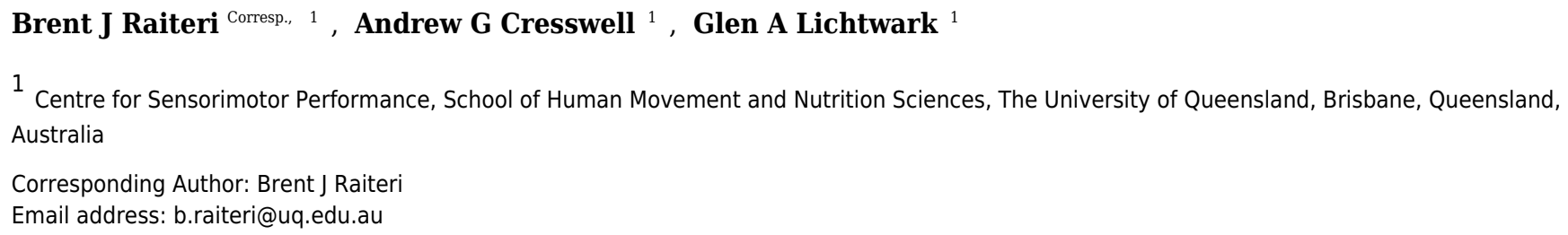

Background. Muscles not only shorten during contraction to perform mechanical work, but they also bulge radially because of the isovolumetric constraint on muscle fibres. Muscle bulging may have important implications for muscle performance, however quantifying three-dimensional (3D) muscle shape changes in human muscle is problematic because of difficulties with sustaining contractions for the duration of an in vivo scan. Although two-dimensional ultrasound imaging is useful for measuring local muscle deformations, assumptions must be made about global muscle shape changes, which could lead to errors in fully understanding the mechanical behaviour of muscle and its surrounding connective tissues, such as aponeurosis. Therefore, the aims of this investigation were a) to determine the intra-session reliability of a novel 3D ultrasound (3DUS) imaging method for measuring in vivo human muscle and aponeurosis deformations and b) to examine how contraction intensity influences in vivo human muscle and aponeurosis strains during isometric contractions. Methods. Participants $(n=12)$ were seated in a reclined position with their left knee extended and ankle at $90^{\circ}$ and performed isometric dorsiflexion contractions up to $50 \%$ of maximal voluntary contraction. 3DUS scans of the tibialis anterior (TA) muscle belly were performed during the contractions and at rest to assess muscle volume, muscle length, muscle cross-sectional area, muscle thickness and width, fascicle length and pennation angle, and central aponeurosis width and length. The 3DUS scan involved synchronous B-mode ultrasound imaging and 3D motion capture of the position and orientation of the ultrasound transducer, while successive cross-sectional slices were captured by sweeping the transducer along the muscle. Results. 3DUS was shown to be highly reliable across measures of muscle volume, muscle length, fascicle length and central aponeurosis length (ICC $\geq 0.98, C V<1 \%$ ). The TA remained isovolumetric across contraction conditions and progressively shortened along its line of action as contraction intensity increased. This caused the muscle to bulge 
centrally, predominantly in thickness, while muscle fascicles shortened and pennation angle increased as a function of contraction intensity. This resulted in central aponeurosis strains in both the transverse and longitudinal directions increasing with contraction intensity. Discussion. 3DUS is a reliable and viable method for quantifying multidirectional muscle and aponeurosis strains during isometric contractions within the same session. Contracting muscle fibres do work in directions along and orthogonal to the muscle's line of action and central aponeurosis length and width appear to be a function of muscle fascicle shortening and transverse expansion of the muscle fibres, which is dependent on contraction intensity. How factors other than muscle force change the elastic mechanical behaviour of the aponeurosis requires further investigation. 
1 Three-dimensional geometrical changes of the human tibialis anterior muscle and its 2 central aponeurosis measured with three-dimensional ultrasound during isometric

3 contractions

4

5 Brent James Raiteri ${ }^{1}$, Andrew Graham Cresswell ${ }^{1}$ and Glen Anthony Lichtwark ${ }^{1}$

6

$7 \quad{ }^{1}$ The University of Queensland, Centre for Sensorimotor Performance, School of Human

8 Movement and Nutrition Sciences, Brisbane, QLD, Australia

9

10 Corresponding author

11 Brent Raiteri ${ }^{1}$

12 School of Human Movement and Nutrition Sciences, Faculty of Health and Behavioural

13 Sciences, The University of Queensland, Brisbane, QLD, 4072, Australia.

14 Email address: b.raiteri@uq.edu.au

15 


\section{Abstract}

17 Background. Muscles not only shorten during contraction to perform mechanical work, but they also bulge radially because of the isovolumetric constraint on muscle fibres. Muscle bulging may have important implications for muscle performance, however quantifying three-dimensional (3D) muscle shape changes in human muscle is problematic because of difficulties with sustaining contractions for the duration of an in vivo scan. Although two-dimensional ultrasound imaging is useful for measuring local muscle deformations, assumptions must be made about global muscle shape changes, which could lead to errors in fully understanding the mechanical

24 behaviour of muscle and its surrounding connective tissues, such as aponeurosis. Therefore, the 25 aims of this investigation were a) to determine the intra-session reliability of a novel 3D ultrasound (3DUS) imaging method for measuring in vivo human muscle and aponeurosis deformations and b) to examine how contraction intensity influences in vivo human muscle and aponeurosis strains during isometric contractions.

Methods. Participants $(n=12)$ were seated in a reclined position with their left knee extended and ankle at $90^{\circ}$ and performed isometric dorsiflexion contractions up to $50 \%$ of maximal voluntary contraction. 3DUS scans of the tibialis anterior (TA) muscle belly were performed during the contractions and at rest to assess muscle volume, muscle length, muscle cross-sectional area, muscle thickness and width, fascicle length and pennation angle, and central aponeurosis width and length. The 3DUS scan involved synchronous B-mode ultrasound imaging and 3D motion capture of the position and orientation of the ultrasound transducer, while successive crosssectional slices were captured by sweeping the transducer along the muscle.

37 Results. 3DUS was shown to be highly reliable across measures of muscle volume, muscle 38 length, fascicle length and central aponeurosis length ( $\mathrm{ICC} \geq 0.98, \mathrm{CV}<1 \%$ ). The TA remained 
39 isovolumetric across contraction conditions and progressively shortened along its line of action

40 as contraction intensity increased. This caused the muscle to bulge centrally, predominantly in

41 thickness, while muscle fascicles shortened and pennation angle increased as a function of

42 contraction intensity. This resulted in central aponeurosis strains in both the transverse and

43 longitudinal directions increasing with contraction intensity.

44 Discussion. 3DUS is a reliable and viable method for quantifying multidirectional muscle and 45 aponeurosis strains during isometric contractions within the same session. Contracting muscle 46 fibres do work in directions along and orthogonal to the muscle's line of action and central 47 aponeurosis length and width appear to be a function of muscle fascicle shortening and 48 transverse expansion of the muscle fibres, which is dependent on contraction intensity. How 49 factors other than muscle force change the elastic mechanical behaviour of the aponeurosis 50 requires further investigation. 


\section{Introduction}

52 Muscle fibres remain isovolumetric during skeletal muscle contraction (Huxley, 1953; Elliott,

53 Lowy \& Worthington, 1963) and simultaneously shorten along their line of action and bulge

54 radially during active force production (Wakeling \& Randhawa, 2014). Muscle fibre shortening

55 therefore creates transverse and longitudinal forces within the muscle during contraction and

56 may stretch the elastic connective tissues, such as aponeurosis, bi-axially (Azizi \& Roberts,

57 2009). Variations in the amount of fibre shortening and transverse expansion at different

58 contraction forces may change the elastic behaviour of the aponeurosis, by altering its width and

59 length. For instance, the transverse strain of the turkey lateral gastrocnemius aponeurosis has

60 been shown to increase with muscle force up to $20 \%$ of maximum isometric force and

61 subsequently increase the longitudinal stiffness of the aponeurosis by two to three times during

62 isotonic contractions compared to passive stretches (Azizi \& Roberts, 2009). This mechanism for

63 varying series elasticity could have significant implications for our predictions of work output in

64 muscles that rely heavily on elastic strain energy storage and return during stretch-shorten cycles

65 (Wilson \& Lichtwark, 2011).

66

67 Understanding how muscle bulging influences muscle behaviour in human muscles remains

68 problematic, due to difficulties in performing steady submaximal contractions for the length of

69 time it takes to image the entire muscle with in vivo techniques, such as magnetic resonance

70 imaging. To our knowledge, only one investigation has determined in vivo human aponeurosis

71 width and length changes concurrently during contraction. This ultrasound imaging study by

72 Maganaris and colleagues (2001) found an increase in central aponeurosis width and length of

73 the tibialis anterior (TA) muscle during maximal dorsiflexion contractions. However, the 
74 aponeurosis length increase (7\%) was much greater than the length increase $(1.6 \%)$ subsequently

75 estimated in a study by Tilp and colleagues (2012), suggesting that that two-dimensional

76 ultrasound (2DUS) imaging techniques may not be accurate for quantifying aponeurosis strains.

77 Interestingly, the study by Tilp (2012) also found that central aponeurosis length decreased at 78 approximately $10 \%$ of maximal voluntary isometric contraction (MVIC), and while this finding 79 could be due to measurement error, it may indicate that at low activations the shortening of muscle fibres produces a transversal rather than longitudinal aponeurosis strain and a subsequent

81 reduction in aponeurosis length (Azizi \& Roberts, 2009).

82

83 One potential method for more accurately determining both longitudinal and transverse strains of 84 the aponeurosis during contractions is three-dimensional ultrasound (3DUS) imaging (Barber, 85 Barrett \& Lichtwark, 2009). This technique has been used to measure longitudinal and transverse 86 strains of the proximal Achilles tendon during isometric plantar flexion contractions (Farris et 87 al., 2013) and should also be suitable to provide more detailed quantification of how factors such 88 as muscle shortening and bulging influence strains of connective tissue.

The aim of this study was to use a novel freehand, 3DUS imaging technique to measure the in

91 vivo human TA muscle and aponeurosis deformations (defined in Fig. 1) during submaximal 92 isometric dorsiflexion contractions from rest to 50\% MVIC. We first determined the intra93 session reliability of using 3DUS to make measures of muscle volume, muscle length, and 94 aponeurosis width and length at rest and during each submaximal contraction intensity. After 95 assessing the reliability, we tested the hypothesis that the muscle fascicles and muscle belly 96 would shorten while the muscle cross-sectional area (CSA) would increase as a function of 
97 contraction intensity, so that muscle volume would remain relatively constant. We further

98 hypothesized that bulging of the muscle, orthogonal to its line of action, would be driven by

99 progressive increases in muscle thickness from increases in fascicle pennation angle for the low 100 to moderate forces. 


\section{Methods}

102 Participants

103 Twelve participants (age: 24 (mean) \pm 2 (standard deviation) years, height: $175 \pm 7 \mathrm{~cm}$, body

104 mass: $69 \pm 10 \mathrm{~kg}, 6$ males) with no recent (<12 months) history of lower limb injury or surgery

105 and no preexisting neuromuscular disorders, volunteered to participate in the study. All

106 participants were recreationally active at the time of testing and provided written informed

107 consent prior to participation. The study protocol was conducted in accordance with the

108 Declaration of Helsinki and approved (HMS14/0704) and endorsed by the University of

109 Queensland Human Research Ethics Committee (HREC).

110

111 Experimental setup and design

112 Participants were seated in a reclined position with their hips at $130^{\circ}$ and the plantar aspect of

113 their left foot flush against a custom-built rigid footplate (Fig. 2). The left knee was fully

114 extended and the left ankle was at $90^{\circ}$ (base of foot approximately perpendicular to shank).

115 Padded wooden blocks were positioned between the superior dorsal aspect of the left foot and

116 the frame housing the foot to minimize ankle rotation during dorsiflexion contractions.

118 Prior to testing, participants performed five voluntary submaximal isometric dorsiflexion

119 contractions (1-s hold, 1 -s rest, $\sim 80 \%$ of maximum) to precondition the muscle-tendon unit

120 (Maganaris, Baltzopoulos \& Sargeant, 2002). Following this, two maximal isometric

121 dorsiflexion contractions (3-s holds) were performed. In some cases additional maximal

122 contractions were performed until there was no more than $5 \%$ difference in the maximum force 123 attained. 
124

125 Participants performed at least two $\sim 40$-s isometric dorsiflexion contractions at 5, 10, 25 and

$12650 \%$ MVIC in a randomised order. Pilot testing revealed that the 50\% MVIC level was the

127 maximum that could be sustained without a drop in torque for a 40 -s period. Live visual

128 feedback of the dorsiflexion force relative to time was provided via a monitor to enable subjects

129 to match superimposed submaximal force levels. 3DUS scans of the entire TA muscle belly were

130 performed during the contractions and at rest to assess muscle volume, muscle belly length,

131 muscle CSA, muscle thickness and width, fascicle length and pennation angle, and central

132 aponeurosis width and length. At least 1-min of rest was provided between contractions to

133 minimize any possible fatigue effects. Subjects were also asked and given more rest if required.

134 Two scans were analysed separately from each condition to assess the intra-session repeatability

135 of the measures.

136

137 Three-dimensional ultrasound

138 3DUS has been shown to be a valid and reliable technique for the in vivo measurement of human

139 gastrocnemius muscle belly length and volume while muscles are relaxed (Barber, Barrett \&

140 Lichtwark, 2009). The 3DUS scan involved B-mode ultrasound imaging (SonixTouch,

141 Ultrasonix, BC, Canada) with synchronous tracking of the ultrasound transducer position and

142 orientation. A four-camera optical motion analysis system recording at $120 \mathrm{~Hz}$ (Optitrack,

143 Natural Point, OR, USA) was used to track the position and orientation of the ultrasound

144 transducer by tracking the 3D position of four spherical reflective markers (9.5 mm diameter)

145 that were rigidly attached to the transducer. Conventional B-mode ultrasound images of the TA

146 muscle were acquired with a single transverse sweeping scan from the proximal to distal or distal 
147 to proximal end of the muscle belly and were subsequently transformed into the global

148 coordinate system using Stradwin software (v5.0, Mechanical Engineering, Cambridge

149 University, UK). Prior to scanning, the system was temporally and spatially calibrated using the

150 single wall phantom calibration protocol available in the Stradwin software (Prager et al., 1998),

151 as has been described elsewhere (Barber, Barrett \& Lichtwark, 2009).

152

153 Transverse images of the soft tissues within the lower left leg were recorded at approximately

154 10-15 frames/s using a 60 mm linear transducer (L14-5W/60 Linear, Ultrasonix, BC, Canada)

155 with a central frequency of $10 \mathrm{MHz}$ and maximal depth of $55 \mathrm{~mm}$. A 2-cm thick echogenic

156 ultrasound gel pad (Aquaflex, Parker Laboratories, NJ, USA) was positioned between the

157 transducer and the skin to conform to the shape of the leg when light pressure was applied,

158 ensuring that adequate contact was made across the skin so that transverse images of the TA

159 muscle contained visible muscle borders. Echogenic gel was also applied over the skin to reduce

160 friction during the sweeping scan. The total scan time was $\sim 35$-s and the average distance

161 between frames was $\sim 1 \mathrm{~mm}$.

162

163 Force measurements

164 Net ankle force was determined using a custom-built rigid footplate with a force transducer 165 attached close to the centre of the forefoot. While the foot was constrained, contraction of the 166 dorsiflexor muscles resulted in only small rotations at the ankle $\left(<5^{\circ}\right.$ as determined previously 167 during dorsiflexion MVICs using an identical experimental setup (Raiteri, Cresswell \& 168 Lichtwark, 2015)). Muscle and aponeurosis length changes and changes in the centre of pressure 
169 due to ankle rotation were therefore not accounted for as they were considered to be negligible.

170 The force signal was amplified 500 times and sampled at $1 \mathrm{kHz}$.

171

172 Data analysis

173 Muscle CSA was manually segmented from sequential transverse images of the 3DUS scans at

$1745-15 \mathrm{~mm}$ intervals from approximately the level of the tibial tuberosity to $\sim 3 \mathrm{~cm}$ proximal to the

175 level of the ankle malleoli. The same operator (BR) performed the segmentations across all scans

176 in a randomised order on at least three separate occasions. The final segmentations were

177 inspected in a reconstructed sagittal plane image re-slice of the muscle belly, where the plane

178 could be translated in a medial-lateral direction relative to the line of action of the muscle. A

179 very low resolution shape-based paradigm (Treece et al., 2000) was used to interpolate a surface

180 through the segmented slices and algorithms available in Stradwin were used to turn the muscle

181 cross-sections into a smoothed 3D triangle-based model (Treece, Prager \& Gee, 1999) to

182 calculate muscle volume (Treece et al., 1999). The 3D mesh models were then exported for post-

183 processing in Matlab (R2014b, MathWorks, MA, USA).

184

185 Muscle and central aponeurosis lengths were calculated as the straight-line distance between the

186 most proximal and distal landmark locations in 3D space, relative to the laboratory coordinate

187 frame. These landmarks were positioned in the approximate centre of the muscle or central

188 aponeurosis in the first and last transverse slices where the structure was visible and were

189 identified on at least two separate occasions. The landmark positions were confirmed in a

190 reconstructed sagittal plane image of the muscle belly where the muscle and central aponeurosis

191 end points were clear. The central aponeurosis was manually outlined along its width medially to 
192 laterally in the transverse images using landmarks that were spaced sequentially at $10-15 \mathrm{~mm}$

193 intervals along the length of the central aponeurosis. This was performed at least two times for

194 each 3DUS scan and the order that scans were analysed was randomised. The 3D position of

195 each central aponeurosis landmark within the laboratory reference frame was later exported for 196 post-processing in Matlab.

197

198 A weighted principal component analysis (PCA) was performed in Matlab to determine the 199 longitudinal axis of the muscle and the central aponeurosis separately. Before the PCA could be 200 performed for the central aponeurosis, a triangular mesh was first reconstructed from the 3D 201 position data based on a Delaunay algorithm (Image Processing Toolbox; Matlab, Mathworks, 202 R2014b, Natick, MA). The PCA involved subtracting the weighted mean (using the areas of 203 triangles in the 3D triangular mesh as weights) from each of the data dimensions and calculating 204 the eigenvectors and eigenvalues of the weighted covariance matrix. Then, a feature vector (x, $\mathrm{y}$ 205 and z) was constructed from the eigenvectors that explained the greatest variance in the data in a 206 descending order so that the long axis corresponded to the $\mathrm{x}$-axis and each surface reconstruction 207 (muscle or central aponeurosis) was transformed and rotated accordingly.

209 Following the PCA, the mesh vertices of the muscle reconstructions were down-sampled by a 210 factor of 10 and a triangulated tight surface was returned based on a Crust algorithm (Luigi, 211 2008). The mesh normals of both the muscle and central aponeurosis reconstructions were then 212 unified and their feature vectors replicated to produce the coordinates of a 3D rectangular grid. 213 From there, the muscle CSA and central aponeurosis width in the transverse plane (y-z plane) 214 along the $\mathrm{x}$-axis was calculated (Fig. 3A\&B) at 1\% increments of the total length from the 
215 centroid. This process essentially re-sliced the volume in the y-z plane of the PCA axis system to

216 ensure that there was no distortion due to the angle of the images that were taken relative to the

217 muscle. To determine muscle thickness and width, the muscle surface reconstruction was

218 transformed and rotated into the $\mathrm{x}, \mathrm{y}$ and z-axes calculated by the PCA of the central aponeurosis

219 from the respective contraction condition. Thickness and width of the PCA-transformed data in

220 the $y-z$ plane was calculated at the centre of the muscle slice at $1 \%$ increments of the total muscle

221 belly length from the centroid. Muscle thickness was defined as the vertical distance of the

222 muscle slice in the $y$-dimension and muscle width as the horizontal distance of the muscle slice

223 in the z-dimension (Fig. 3A).

224

225 Fascicle length and pennation angle were measured at different regions of the muscle using a

226 reconstructed sagittal plane image re-slice centered on the most proximal location of the central

227 aponeurosis in 3D space. The final sagittal plane image re-slice for measuring fascicle length and

228 pennation angle for one 3DUS scan was determined visually by rotating the image plane

229 clockwise or anti-clockwise along the horizontal axis until the image displayed the clearest and

230 most continuous muscle fascicles, which was presumed to correspond to the plane of muscle

231 fascicles (Narici et al., 1996). Three fascicles and their corresponding pennation angles (the

232 angle of fascicle insertion into the central aponeurosis) were then measured in both the

233 superficial and deep compartments of the muscle at proximal, middle and distal ends of the

234 central aponeurosis (Fig. 3C). Because the fascicle curvature at rest and during contraction was

235 small (e.g. Fig. 3C), which has been reported previously (Maganaris \& Baltzopoulos, 1999) we

236 felt that using a straight line to estimate fascicle length was an acceptable approximation of true 237 fascicle length. 


\section{Statistics}

240 Muscle segmentations were not performed on one subject due to difficulties in observing the

241 lateral and deep borders of the TA muscle ( $\mathrm{n}=11$ for muscle volume and muscle CSA measures).

242 Central aponeurosis reconstructions from five subjects were excluded from analyses because of

243 difficulties in either visualising the central aponeurosis or unreliable reconstructions because of

244 high curvature and/or irregular shapes of the aponeurosis $(\mathrm{n}=7)$. Muscle thickness and width

245 measures were therefore performed on six individuals because one set of central aponeurosis

246 reconstructions was performed on the participant with no corresponding muscle segmentations.

247 Statistical analyses were performed using commercially available software (Prism 6, La Jolla,

$248 \mathrm{CA}$ ) and the level of significance was set at $P \leq 0.05$. All data are presented as means \pm standard

249 deviation in the text and means \pm standard error in the figures.

250

251 To determine the repeatability of the length and pennation angle measures within the same

252 session, the intraclass correlation coefficient (ICC), the standard error of measurement (SEM)

253 and the coefficient of variation (CV) were calculated. The coefficient of multiple correlation

254 (CMC) was used to assess the intra-session repeatability of muscle CSA, thickness and width,

255 and central aponeurosis width measures. Data were normally distributed according to Shapiro-

256 Wilk normality tests. One-way repeated-measures ANOVAs were used to assess differences in

257 muscle belly and central aponeurosis length changes, central aponeurosis width changes at 50\%

258 of aponeurosis length and muscle CSA, width and thickness changes at $50 \%$ of muscle belly

259 length across the contraction conditions. Muscle fascicle length and pennation angle at rest in the

260 superficial and deep muscle compartments were compared using a paired t-test and Wilcoxon 
261 signed-rank test, respectively. Differences in fascicle length and pennation angle changes

262 between the muscle compartments across the contraction conditions were compared using two-

263 way ANOVAs (contraction intensity $\times$ muscle compartment). 


\section{Results}

265 Reliability of measures

266 TA muscle segmentations for each subject resulted in TA muscle volume varying by only 2-7

$267 \mathrm{~mL}(1-5 \%)$ across the ten segmentations performed at rest through to $50 \% \mathrm{MVIC}$. Muscle

268 volume ranged from 142-284 mL across subjects. The intra-session reliability for muscle belly

269 length, fascicle length and central aponeurosis length measures was excellent at rest and across

270 the contraction conditions (Table 1: $\mathrm{ICC}=0.98->0.99 ; \mathrm{SEM}=0.10-0.78 \mathrm{~mm} ; \mathrm{CV}=0.1-0.6 \%$ ).

271 Notably, the reliability for pennation angle measures was less, particularly measures made within

272 the superficial compartment, as determined by the ICCs of 0.49-0.89. However, the absolute

273 reliability of pennation angle measures was still high (Table 1), as evidenced by the small SEMs

$274\left(\leq 0.66^{\circ}\right)$, and the depressed ICCs were likely to be because of low between-subjects variability in

275 pennation angles rather than increased trial-to-trial variability (Weir, 2005). CMCs for muscle

276 CSA, muscle width and muscle thickness measures indicate that these measures were highly

277 repeatable (range $=0.98->0.99$ ). $\mathrm{CMCs}$ for central aponeurosis widths were slightly lower, but

278 within an acceptable range (range=0.89-0.99). These methods were therefore deemed reliable

279 within the same session and used for additional analysis regarding muscle and aponeurosis shape

280 changes with contraction intensity.

281

282 Muscle shape changes during contraction

283 TA muscle belly length decreased as contraction intensity increased (Fig. 4) and there was a

284 significant main effect of contraction intensity on muscle belly length $(P<0.01)$. Because muscle

285 volume remained relatively constant across contraction conditions as the muscle belly shortened,

286 muscle CSA increased with contraction effort. Peak increases in CSA centred around the middle 
287 of the muscle belly (Fig. 5A). At 50\% of muscle belly length, there was a significant main effect

288 of contraction condition on muscle CSA $(P<0.01$; Table 2$)$. These increases in muscle CSA were

289 mainly driven by increases in muscle thickness rather than muscle width (Fig. 5B\&C). A

290 significant main effect of contraction intensity on muscle thickness was observed at $50 \%$ of

291 muscle belly length $(P<0.01$; Table 2$)$, however the same effect was not found on muscle width

$292(P=0.35 ;$ Table 2).

293

294 Fascicle length and pennation angle changes during contraction

295 TA muscle fascicle length decreased as contraction intensity increased in both the superficial and

296 deep muscle compartments (Fig. 6A), while pennation angle increased (Fig. 6B). The decrease in

297 fascicle length, as a function of contraction intensity, was curvilinear. At rest, the superficial

298 muscle compartment had significantly shorter muscle fascicles than the deep muscle

299 compartment $(68.6 \pm 2.2 \mathrm{~mm}$ and $72.4 \pm 2.5 \mathrm{~mm}$, respectively; $\mathrm{P}<0.01)$, however pennation

300 angle was not significantly different $\left(10.9 \pm 0.57^{\circ}\right.$ and $11.1 \pm 1.5^{\circ}$, respectively; $\left.\mathrm{P}=0.27\right)$. A

301 comparison of fascicle length changes in the superficial and deep muscle compartments across

302 the contraction conditions revealed a significant main effect of contraction intensity $(P<0.01)$,

303 but no effect of muscle compartment $(P=0.15)$ and no significant interaction $(P=0.98)$. The same

304 comparison for pennation angle changes showed significant main effects of both contraction

305 intensity $(P<0.01)$ and muscle compartment $(P<0.01)$ yet no significant interaction $(P=0.94)$.

306

307 Central aponeurosis deformations during contraction

308 There was a significant main effect of contraction intensity on both central aponeurosis width

309 and length $(P<0.01)$. Mean central aponeurosis width decreased slightly at 5\%, although this was 
310 not significantly different from zero. Increases in aponeurosis width were then apparent from 5

311 to $50 \%$ MVIC (Fig. 7A\&B). Central aponeurosis length increased in all of the contraction

312 conditions $(P<0.01$; Fig. $7 \mathrm{C})$. The strains in the transverse direction at $50 \%$ of central

313 aponeurosis length were greater than the strains in the longitudinal direction at the same

314 contraction intensity (Fig. 7B\&C). This was primarily due to differences in the resting length

315 rather than the absolute stretch of the tissue in each direction (Table 3). 


\section{Discussion}

317 The results of this study provide the first evidence of how the human TA muscle bulges and how

318 its central aponeurosis deforms in vivo with increasing contraction intensity during isometric

319 dorsiflexion. The 3DUS technique was suitably reliable and sensitive to make the measures

320 required for assessing muscle shape changes during isometric contraction. The TA muscle belly

321 remained isovolumetric from rest through to 50\% MVIC and progressively shortened along its

322 line of action as contraction intensity increased, which caused the muscle belly to bulge

323 centrally, as expected. This bulging orthogonal to the muscle's line of action presumably

324 occurred because of the transverse strain present in the muscle fibres as they shortened

325 (Wakeling \& Randhawa, 2014) and were constrained to maintain a constant volume (Huxley,

326 1953; Elliott, Lowy \& Worthington, 1963). Fascicle shortening was accompanied by fascicle

327 rotation, and this was permitted by increases in muscle thickness at the centre of the muscle.

328 Muscle fascicle shortening and presumably radial expansion of muscle fibres (Wakeling \&

329 Randhawa, 2014) caused the central aponeurosis to strain in both longitudinal and transverse

330 directions in a curvilinear fashion with contraction intensity within the range tested here.

331

332 The increase in muscle belly shortening observed at higher contraction intensities is not

333 surprising given that muscle fascicle lengths also progressively decreased with contraction

334 intensity at roughly equivalent magnitudes. This indicates that muscle fascicle length changes

335 were likely to be relatively uniform throughout the muscle. Because muscle fibre volume

336 remains relatively constant during contraction (Huxley, 1953; Elliott, Lowy \& Worthington,

337 1963) active fibre shortening must be accompanied by radial expansion of muscle fibres, and

338 these multidirectional forces generated within muscle drive complex changes in muscle shape 
339 (presumably through changes in intramuscular pressures). We found that muscle shortening was

340 accompanied by significant increases in muscle CSA, predominantly around the middle of the

341 muscle. This central muscle bulging was likely to be because the muscle fibres shortened the

342 most in this region (Rahemi, Nigam \& Wakeling, 2014) or because the physiological CSA was

343 greatest in the middle of the muscle and therefore the transverse forces applied to the muscle

344 were likely to be the highest here.

345

346 Increases in muscle CSA were predominantly driven by increases in muscle thickness, rather

347 than muscle width. This finding contradicts results reported by Maganaris and colleagues (2001)

348 who found that TA muscle thickness remains constant during maximal voluntary contraction,

349 and is likely to be because of the different contraction intensities used. The increases in thickness

350 with contraction intensity are perhaps expected when pennation angle increases during

351 contraction, however this also depends on how the muscle bulges (Randhawa \& Wakeling,

352 2015). Data from Azizi and colleagues (2008) showed that muscle thickness significantly

353 decreases with the force of contraction in the lateral gastrocnemius muscle of turkeys, which

354 suggests that lower contraction intensities may favor fast contractions (i.e. the velocity of muscle

355 belly shortening would exceed the velocity of muscle fibre shortening) over forceful

356 contractions. The reason that muscle thickness increases are favoured at lower forces is

357 unknown, but it may be because of increased stiffness of the connective tissue elements in the

358 direction of muscle width compared with muscle thickness (e.g. the relative thickness of the

359 perimysium along muscle fascicles in the direction of width versus thickness may be greater

360 (Sharafi \& Blemker, 2010)). Alternatively, aponeurotic sheets above and below the muscle fibres

361 may permit muscle thickness expansion because they have a low transverse stiffness and strain 
362 more in this direction (potentially due to cross-fibred collagen arrays (Kannus, 2000)) relative to

363 the fascia surrounding the medial and lateral aspects of the TA.

364

365 The muscle thickness increases we observed might also vary from the Maganaris et al. (1999)

366 study because of the different methods used to calculate thickness. Our method of re-slicing the

367 TA muscle in the $y-z$ plane along the longitudinal axis of its central aponeurosis as determined

368 by a PCA enables accurate measures of thickness that might not be possible using a fixed image

369 plane with conventional 2DUS, where it is clear that measurement error is associated with

370 transducer alignment (Bolsterlee, Gandevia \& Herbert, 2016). The fixation of the transducer to

371 the leg in 2DUS may also cause greater muscle compression compared to 3DUS (Wakeling,

372 Jackman \& Namburete, 2013), which may prevent the muscle from increasing in thickness.

373

374 The observation that fascicle rotation increased with fascicle shortening was expected (Narici et

375 al., 1996) and is in agreement with the thickness increases observed in the centre of the muscle

376 (this is where fascicle lengths and pennation angles were primarily measured). Although we did

377 not measure fascicle lengths and pennation angles in the proximal unipennate portion of the

378 muscle or the most distal end, the mean resting pennation angle we observed at rest of $11^{\circ}$ is

379 identical to studies by Maganaris and colleagues (1999), who used 2DUS to image the TA mid-

380 belly, and Hiblar and colleagues (2003), who used 3DUS to measure pennation angles

381 throughout the entire TA muscle belly. The investigation by Hiblar et al. (2003) found that

382 pennation angles in the proximal part of the muscle were greater $\left(15^{\circ}\right)$ compared to pennation

383 angles at the distal end $\left(7^{\circ}\right)$ and that the angle of insertion decreased in the medio-lateral plane

384 towards the boundary of the muscle (where fascicles were curved slightly). Because larger 
385 pennation angles have been associated with greater amounts of fascicle rotation during

386 contraction (Shin et al., 2009), this may allow the longer proximal fascicles (evidenced by

387 greater separation between aponeuroses) to shorten at similar magnitudes and velocities as the

388 distal fascicles during contraction, extending the range that the TA muscle is mechanically

389 efficient. However, because muscle thickness did not increase significantly at the ends of the

390 muscle, it is unlikely that this occurred in our study because pennation angle changes would not

391 have been favored at the proximal end of the TA.

392

393 Fascicle shortening was shown to stretch the central aponeurosis of the TA in a curvilinear

394 manner with respect to contraction intensity. This result has been documented previously at

395 contraction intensities above 10\% MVIC (Maganaris, Kawakami \& Fukunaga, 2001; Tilp, Steib

396 \& Herzog, 2012). However, at contraction intensities of 10\% MVIC, Tilp and colleagues (2012)

397 found that the central aponeurosis length decreased, which is not in line with what we observed

398 at the same contraction intensity. We believe that this difference may be due to difficulties in

399 accurately determining points on the aponeurosis along the longitudinal axis of the muscle when

400 using 2DUS measurements. This may be particularly pertinent at low muscle forces, where there

401 is heterogeneity in force distribution along the aponeurosis (Zuurbier et al., 1994). By using

402 exact end-points of the aponeurosis, we are able to determine a global strain more accurately

403 than in a 2D approach. Our findings suggest that the aponeurosis acts in-series with the

404 contractile element, where it lengthens in proportion to muscle fascicle shortening, at low and

405 moderate forces. Given that the aponeurosis is an elastic material, it therefore likely contributes a

406 significant amount to the storage and return of elastic energy.

407 
408 The curvilinear relationship suggests that stiffness of the aponeurosis increased with force, which

409 is in-line with the known properties of tendinous tissue. A curvilinear relationship between

410 longitudinal aponeurosis stretch and muscle activation has previously been observed in the TA

411 muscle (Maganaris \& Paul, 2000). The relatively large amount of aponeurosis elongation at 5\%

412 and 10\% MVIC may be attributed to the initial extension allowed by the crimp of collagen fibrils

413 (Diamant et al., 1972), which is commonly known as the 'toe-region' (Ker, 1981). At higher

414 activations ( $25 \%$ and $50 \%$ MVIC in our study) the stretch of collagen fibrils in the aponeurosis

415 presumably gave rise to linear increases in aponeurosis length outside of the so-called 'toe'

416 region. A greater stiffness in the aponeurosis at the higher muscle activations reduced muscle

417 fascicle shortening and rotation (i.e. the relationship between fascicle length change / rotation

418 and muscle activation was also curvilinear) for a given change in force output (intensity).

420 Central aponeurosis strains were greater in the transverse direction (14.4\%) compared with the

421 longitudinal direction (4\%) and we believe that this is a function of the material properties of the

422 aponeurosis and a requirement for radial expansion of the muscle fibres. The biaxial stretching of

423 the aponeurosis was probably related to increases in muscle fibre CSA above and below the

424 aponeurosis (Scott \& Loeb, 1995) as fibres maintained a constant volume during shortening

425 (Elliott, Lowy \& Worthington, 1963). The increase in fibre CSA was presumably accounted for

426 by increases in fibre diameter in both the transverse (which was not significant at 5\% MVIC) and

427 sagittal planes, and the stretching of the aponeurosis along these dimensions probably reduced its

428 thickness in the frontal plane. The transverse central aponeurosis strains we measured are in line

429 with previous estimates based on 2DUS imaging (Maganaris, Kawakami \& Fukunaga, 2001;

430 Muraoka et al., 2003). Relative to the longitudinal strains, the transverse aponeurosis strains may 
431 have been greater because of underestimations of central aponeurosis width or because of

432 differences in aponeurosis compliance in the transverse and longitudinal directions. This latter

433 explanation is supported by findings from Azizi and colleagues (2009), who observed a five

434 times smaller transverse elastic modulus compared with the longitudinal elastic modulus in the

435 lateral gastrocnemius aponeuroses of turkeys. The greater stiffness of the aponeurosis in the

436 longitudinal direction was attributed to the primarily longitudinal arrangement of collagen

437 fascicles along the muscle's line of action, while the structures responsible for the low transverse

438 aponeuroses moduli were not confirmed (Azizi, Halenda \& Roberts, 2009). Interestingly,

439 transverse strains in the aponeurosis were later shown to modulate the longitudinal stiffness of

440 the aponeurosis in this muscle preparation (Azizi \& Roberts, 2009). We also observed increases

441 in the longitudinal stiffness of the TA central aponeurosis (curvilinear strain vs intensity

442 relationship), however it is unclear whether this relationship is due to the normal longitudinal

443 properties of tendinous tissue (discussed earlier) or due to biaxial strain causing an increase in

444 longitudinal stiffness.

445

446 It is certainly worth investigating if factors other than muscle force (such as muscle length and

447 activation) can affect the magnitudes of fascicle shortening and subsequent radial expansion of

448 muscle fibres, which in turn may alter transverse aponeurosis strains (Muraoka et al., 2003) and

449 the stiffness of the aponeurosis in the longitudinal direction. Implementing 3DUS to measure

450 aponeurosis strains at different muscle lengths is feasible, provided that an experienced

451 ultrasound operator conducts the scans on a muscle with clearly identifiable borders and an

452 aponeurosis that can be captured with one sweep of the ultrasound transducer. Ideally, joint

453 rotation should be minimised so that aponeurosis length changes are not overestimated due to 
454 passive length changes during contraction and the transducer's orientation about its horizontal

455 and longitudinal axes should be maintained throughout the scan so that the transducer is

456 perpendicular to the muscle for as much of the scan as possible, since deviations in transducer

457 pitch or roll may result in transverse images overlapping and poor image quality that prevents the

458 muscle or aponeurosis from being segmented accurately (we made our statistical comparisons

459 relative to $50 \%$ of muscle and aponeurosis length because we were confident that the transducer

460 was perpendicular to the muscle and aponeurosis here). The use of an ultrasound gel pad

461 between the transducer and the skin would also be helpful for minimising the amount of muscle

462 compression, which in our study we believe was small (we estimate that muscle thickness may

463 have been reduced by $\sim 0.5 \mathrm{~mm}$ at rest due to transducer pressure and to a lesser extent during the

464 isometric contractions because of the higher TA intramuscular pressures (Aratow et al., 1993;

465 Styf et al., 1995)) and therefore unlikely to have altered the main effect of contraction intensity

466 on muscle thickness that we observed.

467

\section{Conclusions}

469 Our results show that 3DUS is a viable and reliable method for quantifying multidirectional

470 muscle and aponeurosis deformations during contractions within the same session and that

471 contraction intensity (i.e. force output) is an important factor for determining the magnitude of

472 TA muscle bulging and shortening, as well as the amount that the central aponeurosis strains in

473 both the longitudinal and transverse directions. It appears that contracting muscle fibres do work

474 in directions along and orthogonal to the muscle's line of action (evidenced by increases in

475 muscle belly shortening and muscle CSA). We have shown that central aponeurosis length and

476 width appear to be a function of muscle fascicle shortening (and presumably subsequent 
477 transverse expansion of muscle fibres), and that there are significant transverse strains, which are

478 likely to indicate transverse loading. How these aponeurosis deformations influence the storage

479 and return of elastic strain energy and if factors other than muscle force change the elastic

480 mechanical behaviour of the aponeurosis requires further investigation. 


\section{References}

482 Aratow M, Ballard RE, Crenshaw AG, Styf J, Watenpaugh DE, Kahan NJ, Hargens AR 1993.

483 Intramuscular pressure and electromyography as indexes of force during isokinetic exercise.

$484 \quad$ Journal of Applied Physiology 74:2634-2640.

485 Azizi E, Roberts TJ 2009. Biaxial strain and variable stiffness in aponeuroses. Journal of

486 Physiology 587:4309-4318. DOI: 10.1113/jphysiol.2009.173690.

487 Azizi E, Brainerd EL, Roberts TJ 2008. Variable gearing in pennate muscles. Proceedings of the

488

489

National Academy of Sciences of the United States of America 105:1745-1750. DOI:

490

10.1073/pnas.0709212105.

Azizi E, Halenda GM, Roberts TJ 2009. Mechanical properties of the gastrocnemius aponeurosis

491 in wild turkeys. Integrative and Comparative Biology 49:51-58. DOI: 10.1093/icb/icp006.

492

Barber L, Barrett R, Lichtwark G 2009. Validation of a freehand 3D ultrasound system for

493

494 morphological measures of the medial gastrocnemius muscle. Journal of Biomechanics 42:1313-1319. DOI: 10.1016/j.jbiomech.2009.03.005.

495

Bolsterlee B, Gandevia SC, Herbert RD 2016. Ultrasound imaging of the human medial

496 gastrocnemius muscle: How to orient the transducer so that muscle fascicles lie in the image

497 plane. Journal of Biomechanics. DOI: 10.1016/j.jbiomech.2016.02.014.

Diamant J, Keller A, Baer E, Litt M, Arridge RGC 1972. Collagen; ultrastructure and its relation 499 to mechanical properties as a function of ageing. Proceedings of the Royal Society B

500 180:293-315. DOI: 10.1098/rspb.1972.0019.

501 Elliott GF, Lowy J, Worthington CR 1963. An X-ray and light-diffraction study of the filament 502 lattice of striated muscle in the living state and in rigor. Journal of Molecular Biology 6:295503 305. DOI: 10.1016/S0022-2836(63)80090-X. 
504 Farris DJ, Trewartha G, McGuigan MP, Lichtwark GA 2013. Differential strain patterns of the

505 human Achilles tendon determined in vivo with freehand three-dimensional ultrasound

506 imaging. The Journal of Experimental Biology 216:594-600. DOI: 10.1242/jeb.077131.

507 Hiblar T, Bolson EL, Hubka M, Sheehan FH, Kushmerick MJ 2003. Three dimensional

508 ultrasound analysis of fascicle orientation in human tibialis anterior muscle enables analysis

509 of macroscopic torque at the cellular level. Advances in Experimental Medicine and Biology

510 538:635-644. DOI: 10.1007/978-1-4419-9029-7_56.

511 Huxley HE 1953. X-Ray analysis and the problem of muscle. Proceedings of the Royal Society B 512 141:59-62. DOI: 10.1098/rspb.1953.0017.

513 Kannus P 2000. Structure of the tendon connective tissue. Scandinavian Journal of Medicine and $514 \quad$ Science in Sports 10:312-320.

515 Ker RF 1981. Dynamic tensile properties of the plantaris tendon of sheep (Ovis aries). The 516 Journal of Experimental Biology 93:283-302.

517 Maganaris CN, Baltzopoulos V 1999. Predictability of in vivo changes in pennation angle of 518 human tibialis anterior muscle from rest to maximum isometric dorsiflexion. European 519 Journal of Applied Physiology and Occupational Physiology 79:294-297. DOI:

$520 \quad 10.1007 / \mathrm{s} 004210050510$.

521 Maganaris CN, Paul JP 2000. Load-elongation characteristics of in vivo human tendon and $522 \quad$ aponeurosis. The Journal of Experimental Biology 203:751-756.

523 Maganaris CN, Baltzopoulos V, Sargeant AJ 2002. Repeated contractions alter the geometry of 524 human skeletal muscle. Journal of Applied Physiology 93:2089-2094. DOI:

$525 \quad$ 10.1152/japplphysiol.00604.2002.

526 Maganaris CN, Kawakami Y, Fukunaga T 2001. Changes in aponeurotic dimensions upon 
muscle shortening: In vivo observations in man. Journal of Anatomy 199:449-456.

528 Muraoka T, Muramatsu T, Kanehisa H, Fukunaga T 2003. Transverse strain of aponeurosis in

529 human tibialis anterior muscle at rest and during contraction at different joint angles. Journal

$530 \quad$ of Applied Biomechanics 19:39-48.

531 Narici MV, Binzoni T, Hiltbrand E, Fasel J, Terrier F, Cerretelli P 1996. In vivo human

532 gastrocnemius architecture with changing joint angle at rest and during graded isometric

533 contraction. Journal of Physiology 496:287-297.

534 Prager RW, Rohling RN, Gee AH, Berman L 1998. Rapid calibration for 3-D freehand

535 ultrasound. Ultrasound in Medicine and Biology 24:855-869.

536 Rahemi H, Nigam N, Wakeling JM 2014. Regionalizing muscle activity causes changes to the

537 magnitude and direction of the force from whole muscles — a modeling study. Frontiers in

538 Physiology 5:298. DOI: 10.3389/fphys.2014.00298.

539 Raiteri BJ, Cresswell AG, Lichtwark GA 2015. Ultrasound reveals negligible cocontraction

540 during isometric plantar flexion and dorsiflexion despite the presence of antagonist

541 electromyographic activity. Journal of Applied Physiology 118:1193-1199. DOI:

$542 \quad$ 10.1152/japplphysiol.00825.2014.

543 Randhawa A, Wakeling JM 2015. Multidimensional models for predicting muscle structure and

544 fascicle pennation. Journal of Theoretical Biology 382:57-63. DOI:

$545 \quad$ 10.1016/j.jtbi.2015.06.001.

546 Scott SH, Loeb GE 1995. Mechanical properties of aponeurosis and tendon of the cat soleus

547 muscle during whole-muscle isometric contractions. Journal of Morphology 224:73-86.

548 DOI: $10.1002 /$ jmor.1052240109.

549 Sharafi B, Blemker SS 2010. A micromechanical model of skeletal muscle to explore the effects 
550 of fiber and fascicle geometry. Journal of Biomechanics 43:3207-3213. DOI:

$551 \quad$ 10.1016/j.jbiomech.2010.07.020.

552 Shin DD, Hodgson JA, Edgerton VR, Sinha S 2009. In vivo intramuscular fascicle-aponeuroses

553 dynamics of the human medial gastrocnemius during plantarflexion and dorsiflexion of the 554 foot. Journal of Applied Physiology 107:1276-1284. DOI:

$555 \quad$ 10.1152/japplphysiol.91598.2008.

556 Styf J, Ballard R, Aratow M, Crenshaw A, Watenpaugh D, Hargens AR 1995. Intramuscular

557 pressure and torque during isometric, concentric and eccentric muscular activity.

558 Scandinavian Journal of Medicine and Science in Sports 5:291-296.

559 Tilp M, Steib S, Herzog W 2012. Length changes of human tibialis anterior central aponeurosis

560 during passive movements and isometric, concentric, and eccentric contractions. European 561 Journal of Applied Physiology 112:1485-1494. DOI: 10.1007/s00421-011-2111-0.

562 Treece GM, Prager RW, Gee AH 1999. Regularised marching tetrahedra: Improved iso-surface 563 extraction. Computers \& Graphics 23:583-598. DOI: 10.1016/S0097-8493(99)00076-X.

564 Treece GM, Prager RW, Gee AH, Berman L 1999. Fast surface and volume estimation from

565 non-parallel cross-sections, for freehand three-dimensional ultrasound. Medical Image

566 Analysis 3:141-173. DOI: 10.1016/S1361-8415(99)80004-8.

567 Treece GM, Prager RW, Gee AH, Berman L 2000. Surface interpolation from sparse cross 568 sections using region correspondence. IEEE Transactions on Medical Imaging 19:1106-

$569 \quad$ 1114. DOI: $10.1109 / 42.896787$.

570 Wakeling JM, Randhawa A 2014. Transverse strains in muscle fascicles during voluntary

571 contraction: A 2D frequency decomposition of B-mode ultrasound images. International

$572 \quad$ Journal of Biomedical Imaging 2014:1-9. DOI: 10.1155/2014/352910. 
573 Wakeling JM, Jackman M, Namburete AI 2013. The effect of external compression on the

574 mechanics of muscle contraction. Journal of Applied Biomechanics 29:360-364.

575 Weir JP 2005. Quantifying test-retest reliability using the intraclass correlation coefficient and

576 the SEM. Journal of Strength and Conditioning Research 19:231-240. DOI:

$577 \quad 10.1519 / 15184.1$.

578 Wilson A, Lichtwark G 2011. The anatomical arrangement of muscle and tendon enhances limb 579 versatility and locomotor performance. Philosophical Transactions of the Royal Society B $580 \quad 366: 1540-1553$. DOI: $10.1098 /$ rstb.2010.0361.

581 Zuurbier CJ, Everard AJ, van der Wees P, Huijing PA 1994. Length-force characteristics of the 582 aponeurosis in the passive and active muscle condition and in the isolated condition. Journal 583 of Biomechanics 27:445-453.

584 


\section{Figure 1}

Schematic representations of the shank in the sagittal plane and the tibialis anterior muscle in the sagittal and transverse planes with its geometrical parameters defined.

Top: A sagittal plane representation of the shank with the tibialis anterior (TA), tibialis posterior (TP), soleus (SOL) and lateral gastrocnemius (LG) muscles identified. Bottom left: A sagittal plane representation of the TA muscle showing its bi-pennate architecture with the superficial and deep muscle fascicles (oblique red lines) at an angle to the aponeuroses (horizontal blue lines) with the free tendon (horizontal black line) in-series with the central aponeurosis. Measures of central aponeurosis length, fascicle length, pennation angle and muscle length have been defined in the sagittal plane. Bottom right: A transverse plane representation of the TA muscle showing its central aponeurosis (horizontal blue line) and the superficial and deep muscle compartments. The ultrasound transducer in the top right schematic identifies the slice location. Measures of central aponeurosis width, muscle thickness and muscle width have been defined and the red shaded area shows muscle crosssectional area (CSA).

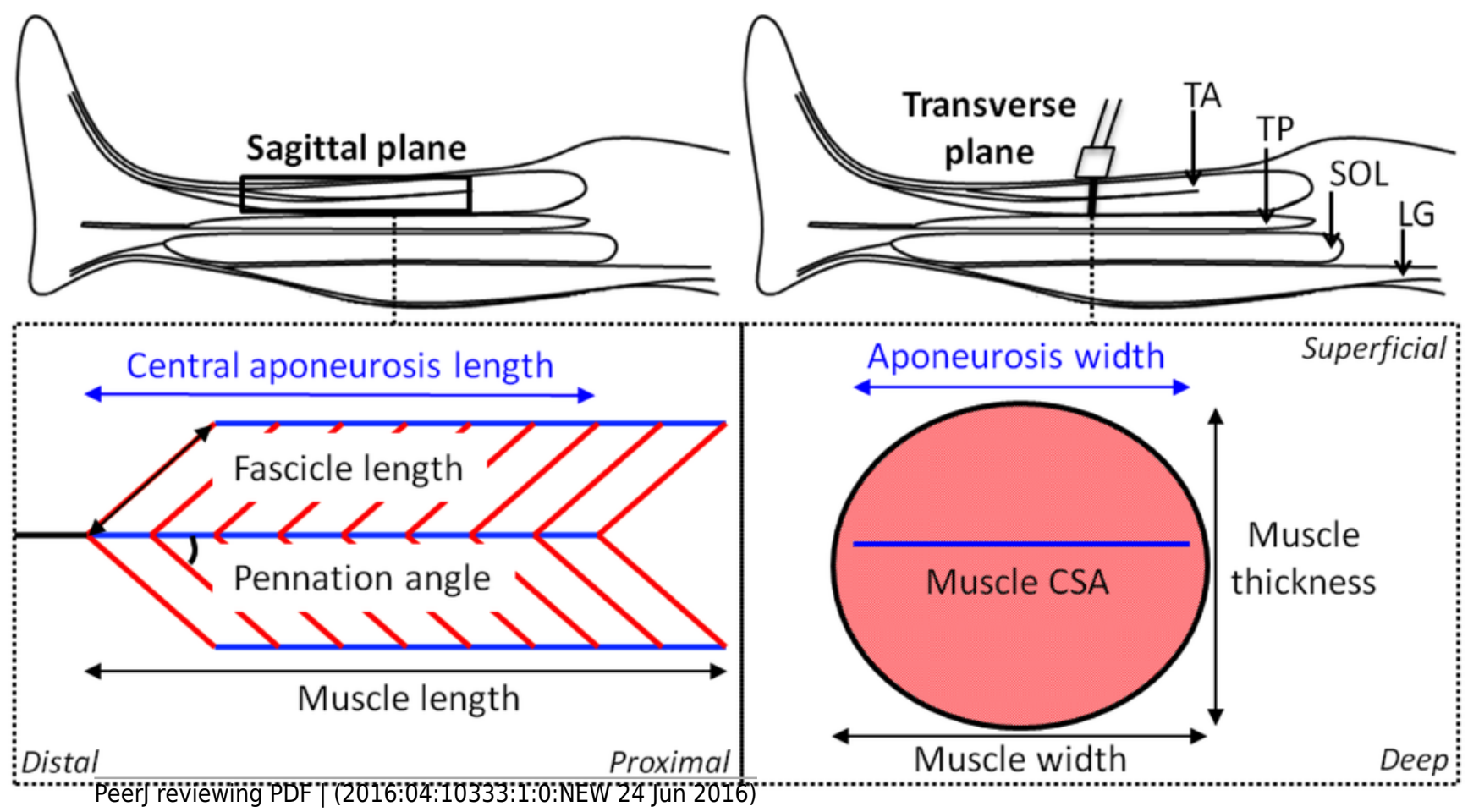


Figure 2

Experimental setup.

A schematic of the footplate and load cell setup and the participant's position during the three-dimensional (3D) ultrasound scans, which combined B-mode imaging of the left-sided tibialis anterior (TA) muscle with 3D motion capture of the ultrasound transducer's position and orientation. Single transverse sweeping scans were performed from the proximal to distal (indicated by the horizontal dotted arrow) or distal to proximal direction at rest and during sustained isometric dorsiflexion contractions.

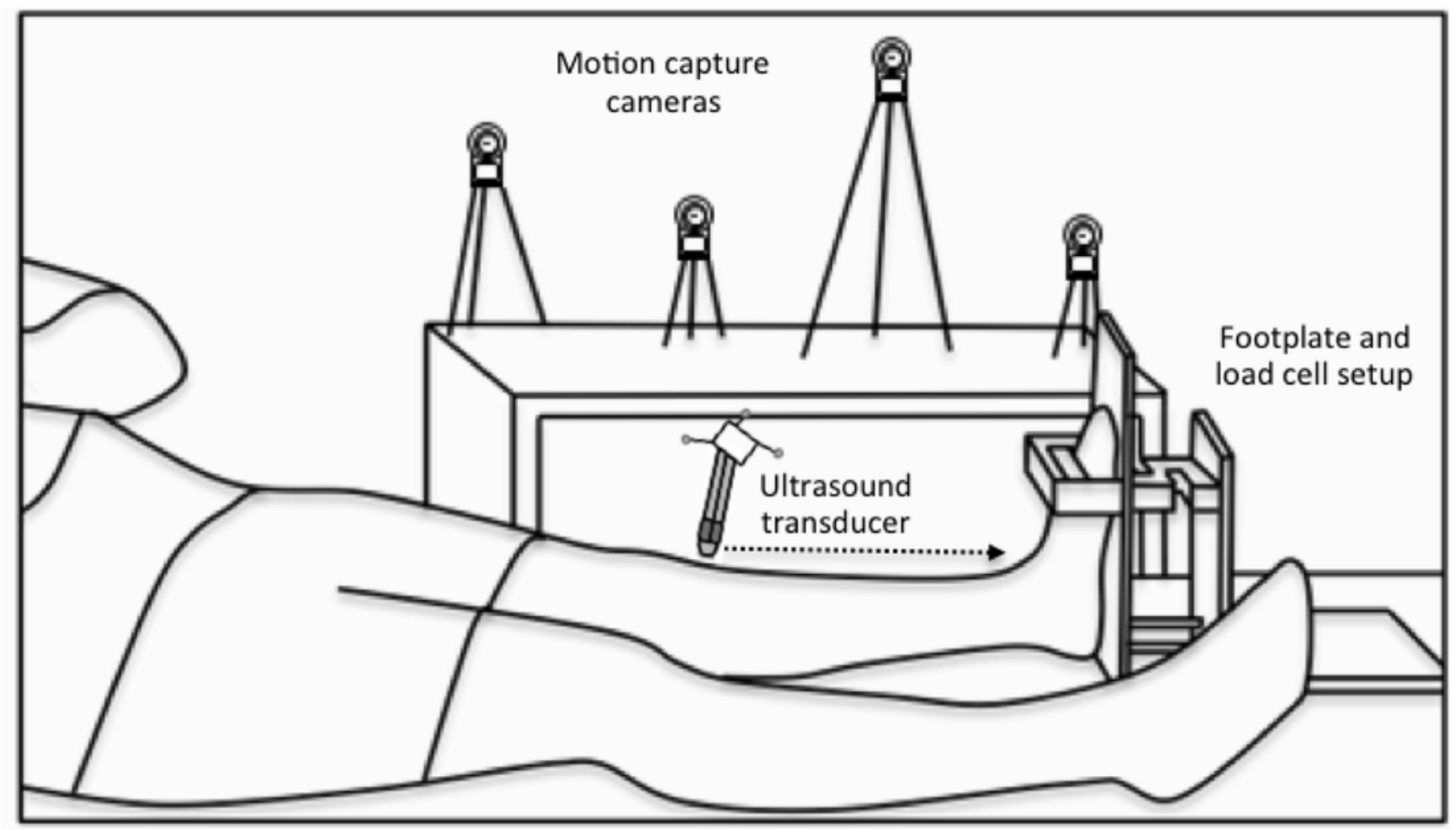




\section{Table $\mathbf{1}$ (on next page)}

Intra-session reliability of tibialis anterior muscle lengths, central aponeurosis lengths, fascicle lengths and pennation angles at rest and as a function of contraction intensity.

${ }_{\mathrm{s}}=$ superficial compartment; ${ }_{\mathrm{d}}=$ deep compartment. 


\begin{tabular}{|c|c|c|c|c|c|}
\hline \multirow{2}{*}{ Intra-class correlation coefficient $t_{2,1}$} & \multicolumn{5}{|c|}{ Contraction intensity (\% MVIC) } \\
\hline & Rest & 5 & 10 & 25 & 50 \\
\hline Muscle length & \multicolumn{5}{|c|}{$>0.99$} \\
\hline Central aponeurosis length & \multicolumn{5}{|c|}{$>0.99$} \\
\hline Fascicle length $_{\mathrm{s}}$ & $>0.99$ & 0.99 & $>0.99$ & $>0.99$ & 0.99 \\
\hline Fascicle length $_{d}$ & 0.98 & 0.99 & $>0.99$ & $>0.99$ & 0.99 \\
\hline Pennation angle $_{\mathrm{s}}$ & 0.82 & 0.89 & 0.59 & 0.88 & 0.49 \\
\hline Pennation angle $_{\mathrm{d}}$ & 0.94 & 0.99 & 0.95 & 0.93 & 0.96 \\
\hline Standard error of measurement & Rest & 5 & 10 & 25 & 50 \\
\hline Muscle length (mm) & 0.78 & 0.42 & 0.34 & 0.29 & 0.36 \\
\hline Central aponeurosis length $(\mathrm{mm})$ & 0.26 & 0.41 & 0.43 & 0.41 & 0.36 \\
\hline Fascicle length $_{\mathrm{s}}(\mathrm{mm})$ & 0.18 & 0.10 & 0.14 & 0.17 & 0.18 \\
\hline Fascicle length $_{d}(\mathrm{~mm})$ & 0.41 & 0.26 & 0.17 & 0.12 & 0.18 \\
\hline Pennation angle ${ }_{\mathrm{s}}\left({ }^{\circ}\right)$ & 0.47 & 0.26 & 0.63 & 0.38 & 0.66 \\
\hline Pennation angle $_{\mathrm{d}}\left({ }^{\circ}\right)$ & 0.43 & 0.21 & 0.32 & 0.42 & 0.42 \\
\hline Coefficient of variation (\%) & Rest & 5 & 10 & 25 & 50 \\
\hline Muscle length & 0.20 & 0.10 & 0.10 & 0.10 & 0.10 \\
\hline Central aponeurosis length & 0.20 & 0.20 & 0.30 & 0.20 & 0.20 \\
\hline Fascicle length $_{\mathrm{s}}$ & 0.30 & 0.30 & 0.20 & 0.30 & 0.30 \\
\hline Fascicle length $_{d}$ & 0.60 & 0.40 & 0.20 & 0.20 & 0.30 \\
\hline Pennation angle $_{\mathrm{s}}$ & 2.50 & 2.20 & 5.40 & 3.00 & 4.80 \\
\hline Pennation angle $_{d}$ & 4.80 & 1.40 & 2.40 & 2.90 & 2.60 \\
\hline
\end{tabular}




\section{Figure 3}

Reconstructions of the tibialis anterior muscle belly, central aponeurosis and muscle fascicles at rest.
A) Individual example of a tibialis anterior (TA) muscle belly reconstruction (red) with a transverse cross-section (light blue) of the muscle reconstruction illustrating how muscle thickness and width measures were made. B) Individual example of a TA central aponeurosis reconstruction (dark blue) with a birds-eye view illustrating how central aponeurosis width and length measures were made. Each image in $\boldsymbol{A}$ and $\boldsymbol{B}$ is represented in the coordinate system defined by the principal component analysis of the central aponeurosis. C) Individual example of a reconstructed sagittal plane re-slice image for the TA muscle. The best sagittal plane re-slice image was determined visually as the image that displayed the clearest most continuous muscle fascicles. Three fascicles in the superficial and deep muscle compartments were analysed in the fascicle region outlined. 


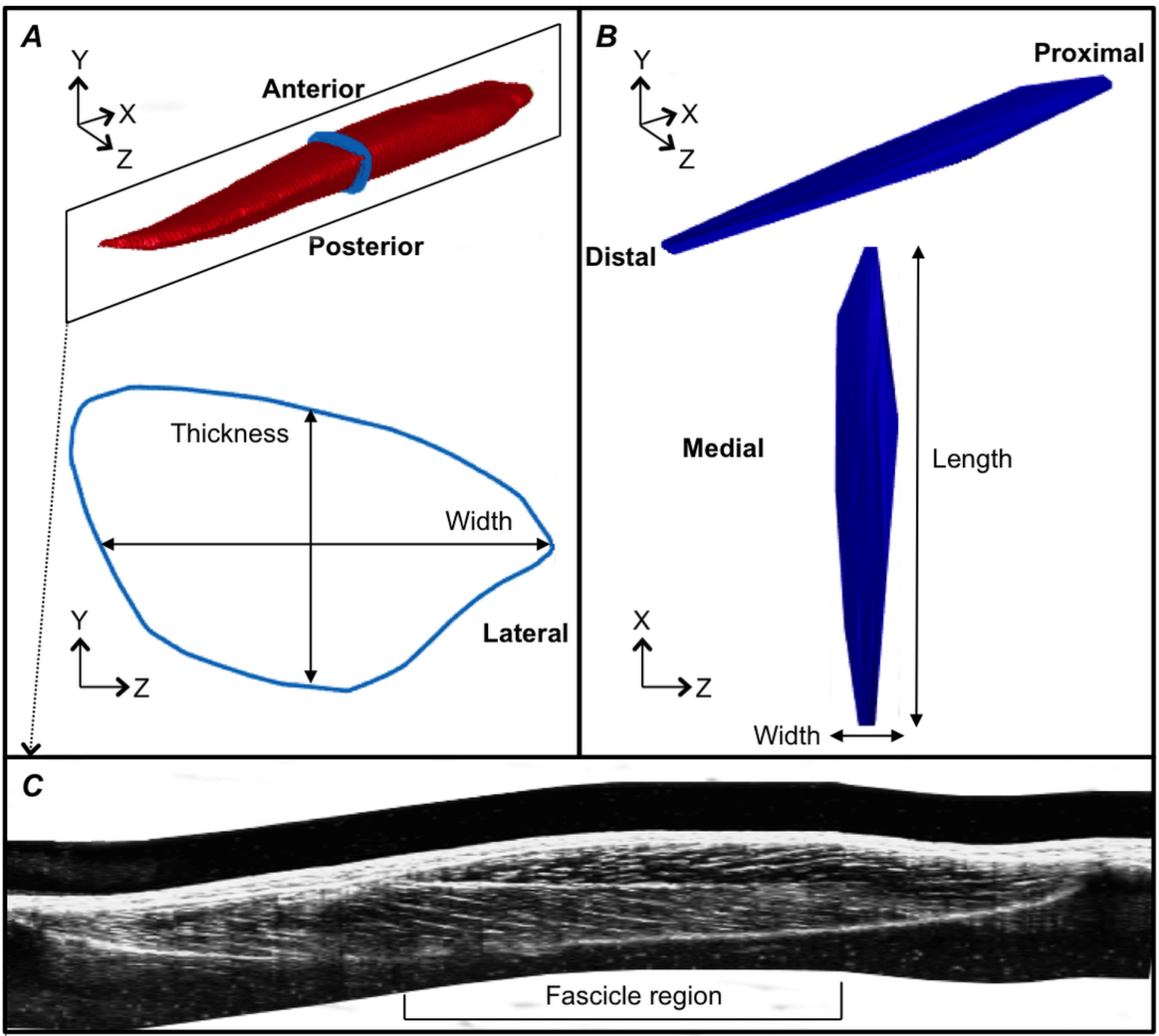


Figure 4

Tibialis anterior muscle length changes during isometric dorsiflexion contractions as a function of contraction intensity.

Negative values indicate shortening. Data are presented as mean \pm standard error.

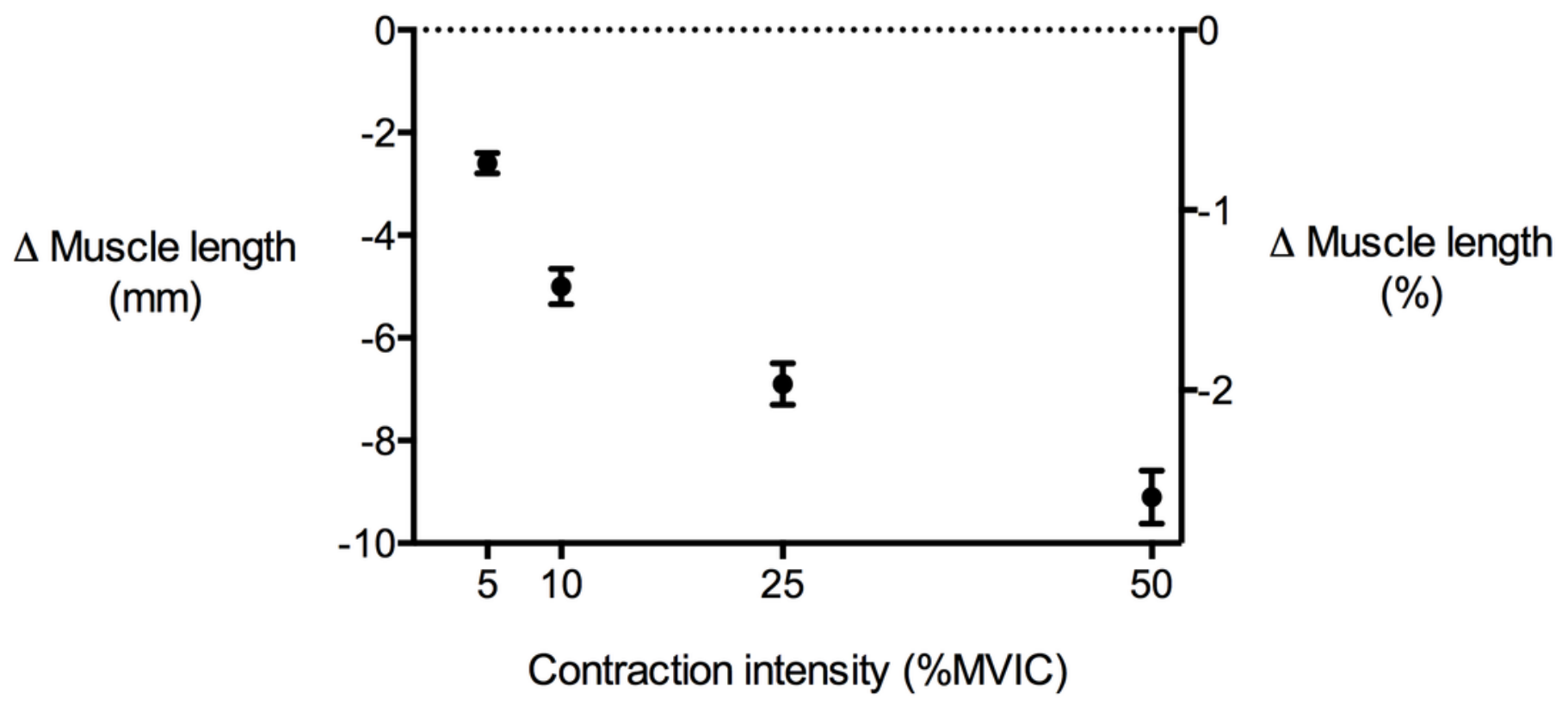




\section{Figure 5}

Tibialis anterior muscle deformations perpendicular to its line of action as a function of contraction intensity.

A) Muscle cross-sectional area (CSA), B) muscle width and C) muscle thickness changes of the tibialis anterior at $25-75 \%$ of muscle length during isometric dorsiflexion contractions at $5 \%, 10 \%, 25 \%$ and $50 \%$ of maximal voluntary isometric contraction. Data are presented as mean \pm standard error. 
A

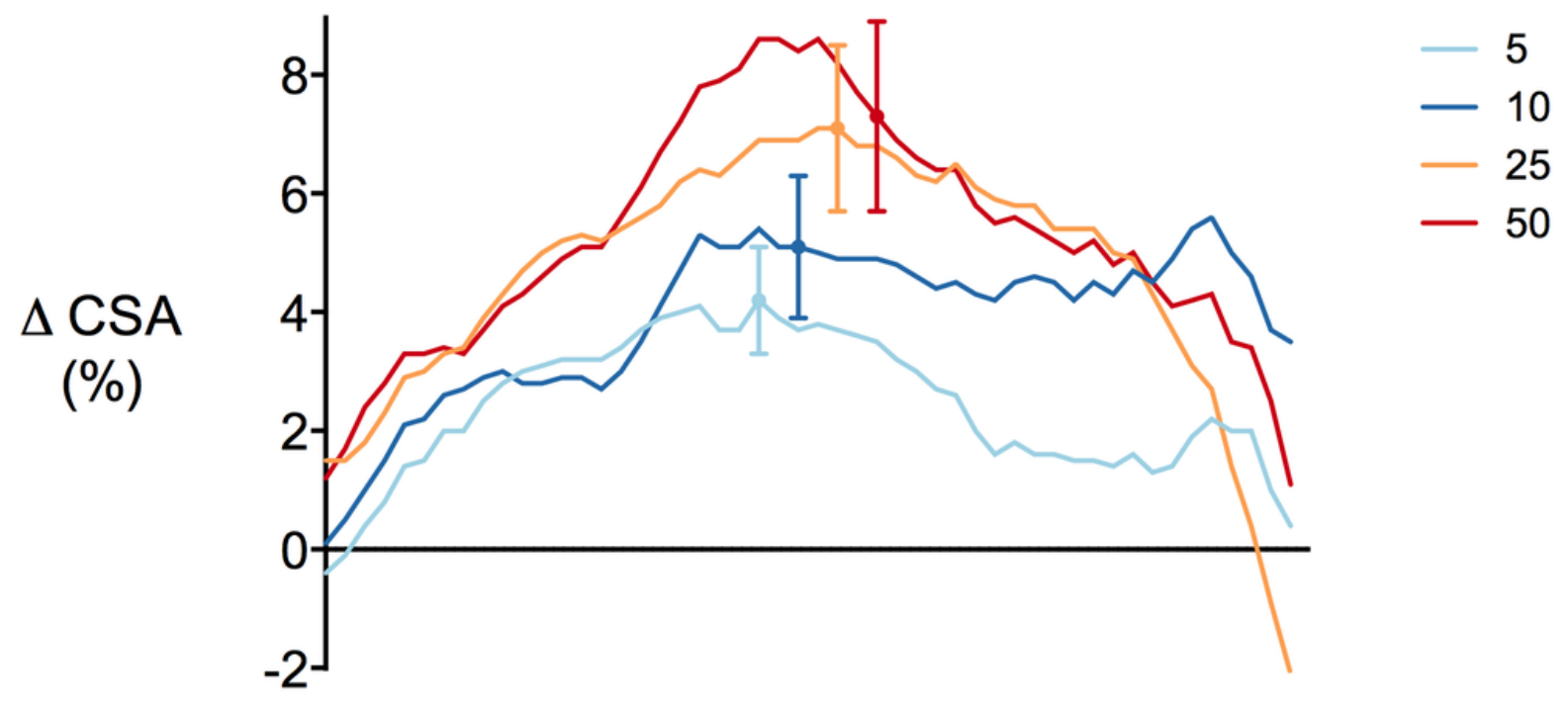

B

$\Delta$ Width

(\%)

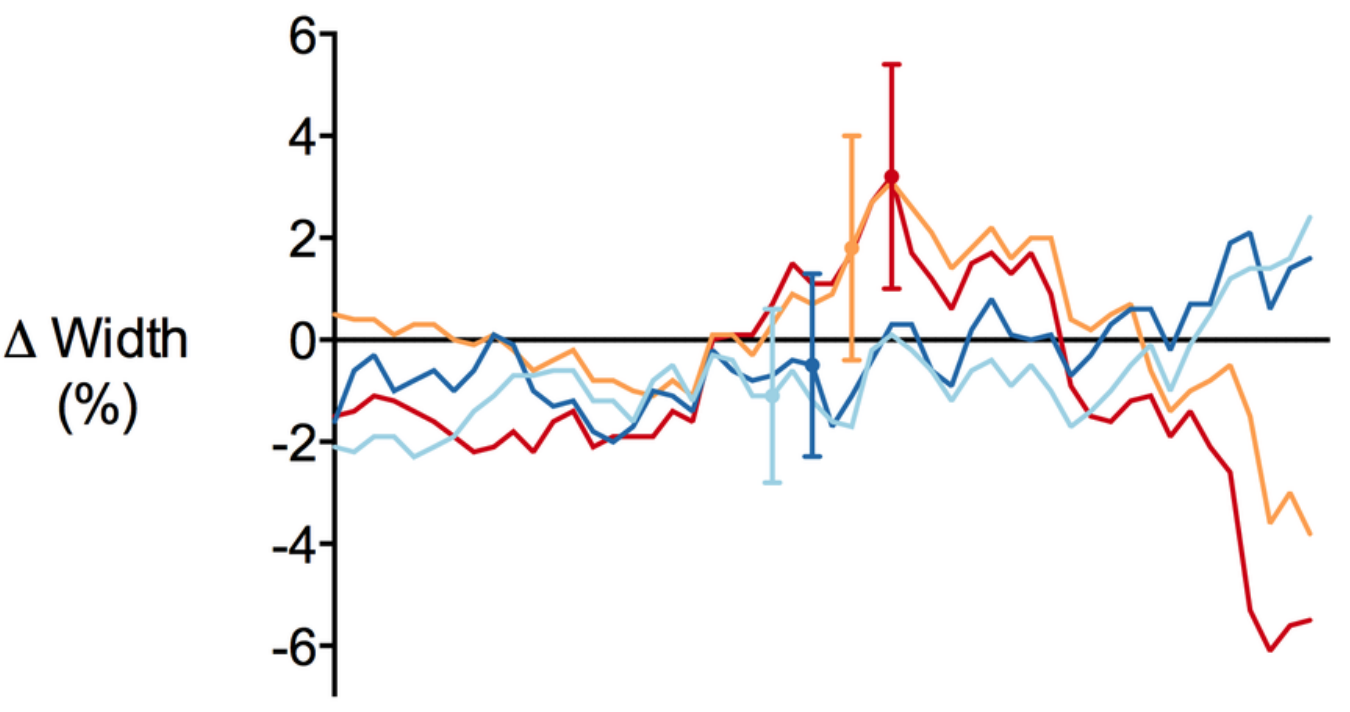

C

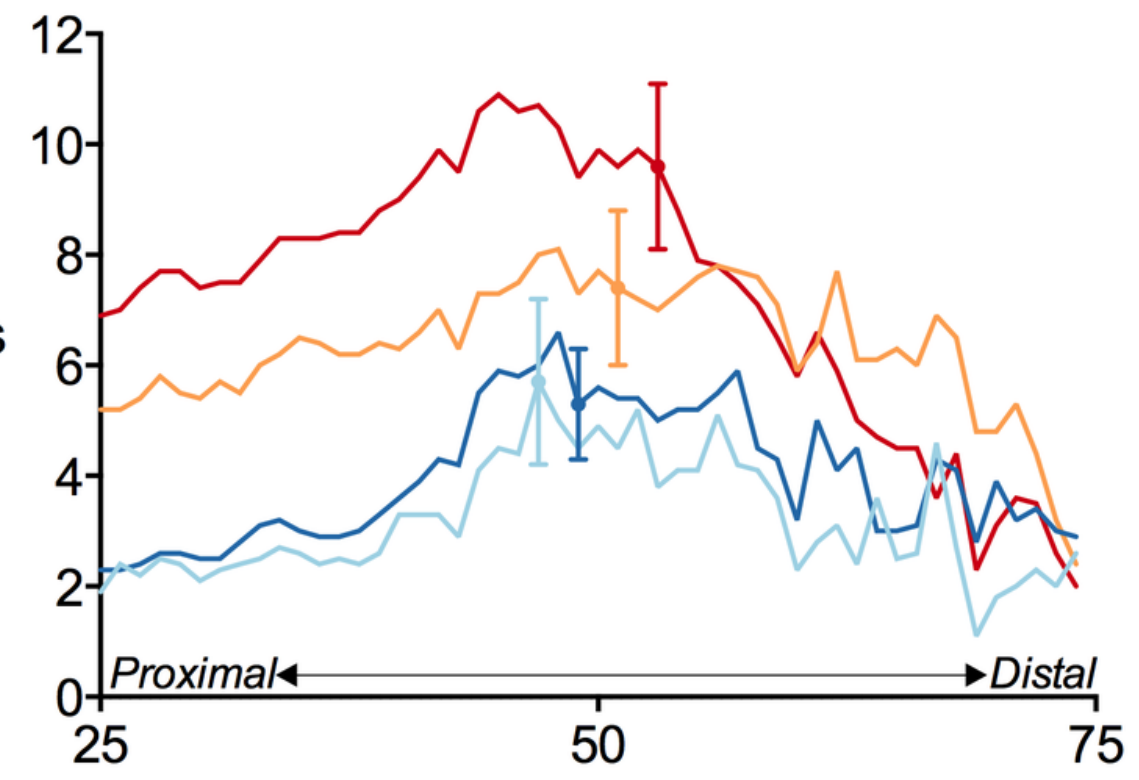

Peer) reviewing PDF | (2016:04:10333:1:0:NEW MAluşie length (\%) 


\section{Table 2 (on next page)}

Changes in tibialis anterior muscle parameters perpendicular to its line of action at 50\% of muscle length as a function of contraction intensity.

MVIC, Maximal Voluntary Isometric Contraction; CSA, Cross-Sectional Area. 
1

\begin{tabular}{|c|c|c|c|c|c|}
\hline \multirow{2}{*}{ Muscle Parameter } & \multicolumn{4}{|c|}{ Contraction Intensity (\% MVIC) } & \multirow{2}{*}{ P value } \\
\cline { 2 - 6 } & $\mathbf{5}$ & $\mathbf{1 0}$ & $\mathbf{2 5}$ & $\mathbf{5 0}$ & \\
\hline$\Delta$ Muscle CSA (mm $\left.{ }^{2}\right)$ & $28.3 \pm 21.6$ & $36.5 \pm 26.3$ & $50.3 \pm 29.7$ & $63.3 \pm 47.7$ & $<0.01$ \\
\hline $\begin{array}{c}\Delta \text { Muscle thickness } \\
(\mathrm{mm})\end{array}$ & $1.2 \pm 1.0$ & $1.4 \pm 0.6$ & $2.0 \pm 0.6$ & $2.5 \pm 0.8$ & $<0.01$ \\
\hline$\Delta$ Muscle width (mm) & $-0.7 \pm 1.9$ & $-0.8 \pm 1.7$ & $0.3 \pm 1.4$ & $0.4 \pm 1.7$ & 0.35 \\
\hline
\end{tabular}

2 
Figure 6

Tibialis anterior muscle architecture changes during isometric dorsiflexion contractions as a function of contraction intensity.

A) Muscle fascicle length and $\boldsymbol{B}$ ) pennation angle changes of the tibialis anterior in the superficial and deep compartments at 5\%, 10\%, 25\% and 50\% of maximal voluntary isometric contraction. Negative values in $\boldsymbol{A}$ indicate shortening. Data are presented as mean \pm standard error.

$\boldsymbol{A}$

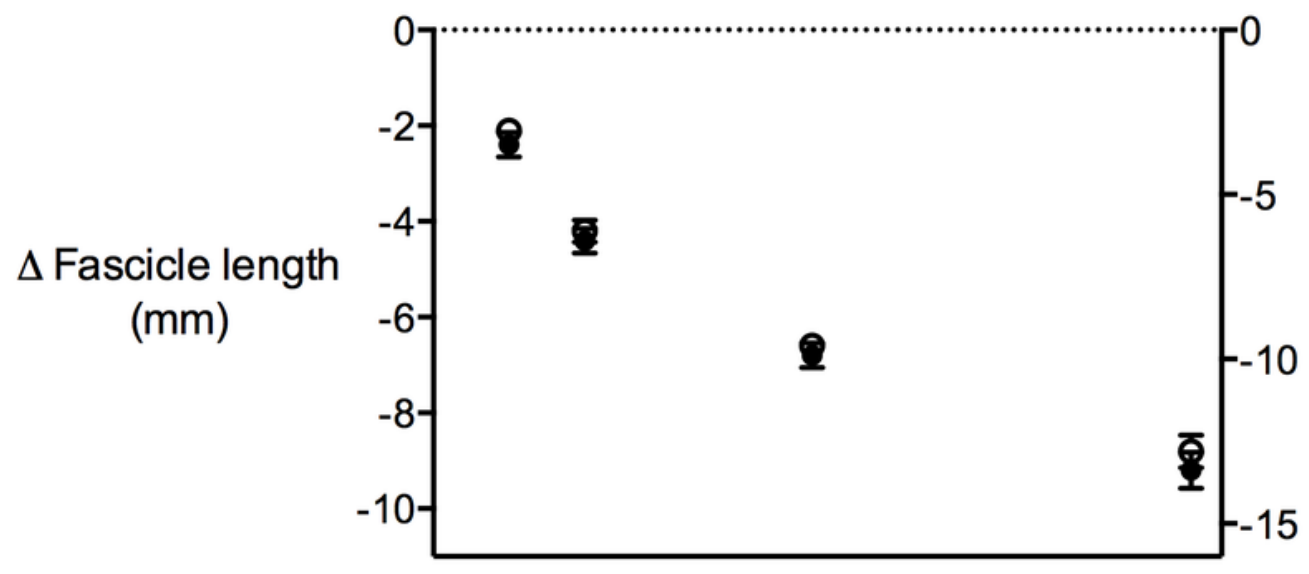

- Superficial

- Deep

$\Delta$ Fascicle length

(\%)

B

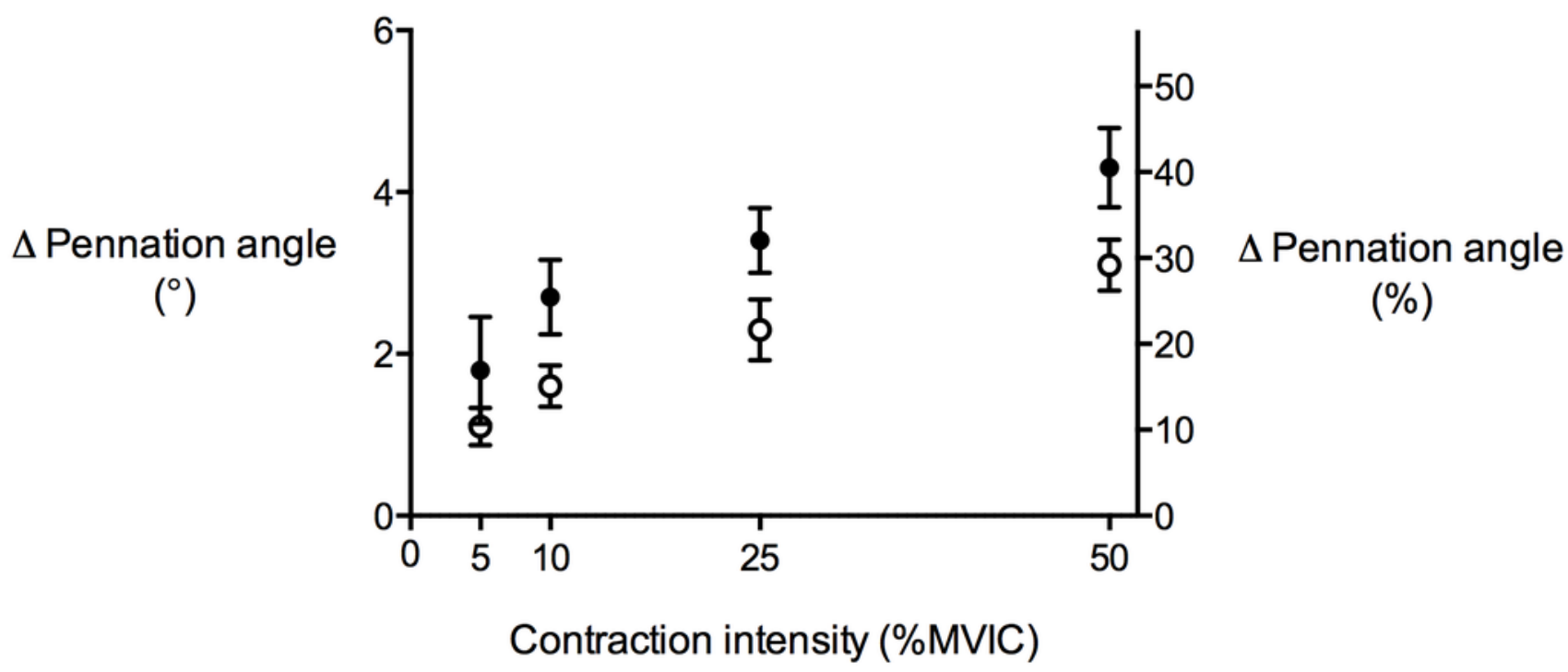




\section{Figure 7}

Tibialis anterior central aponeurosis deformations during isometric dorsiflexion contractions as a function of contraction intensity.

A) Central aponeurosis (CA) width changes of the tibialis anterior at $10-90 \%$ of central aponeurosis length, $\boldsymbol{B}$ ) central aponeurosis width changes at $50 \%$ of central aponeurosis length and $\mathbf{C}$ ) central aponeurosis length changes at $5 \%, 10 \%, 25 \%$ and $50 \%$ of maximal voluntary isometric contraction. Data are presented as mean \pm standard error. 
A

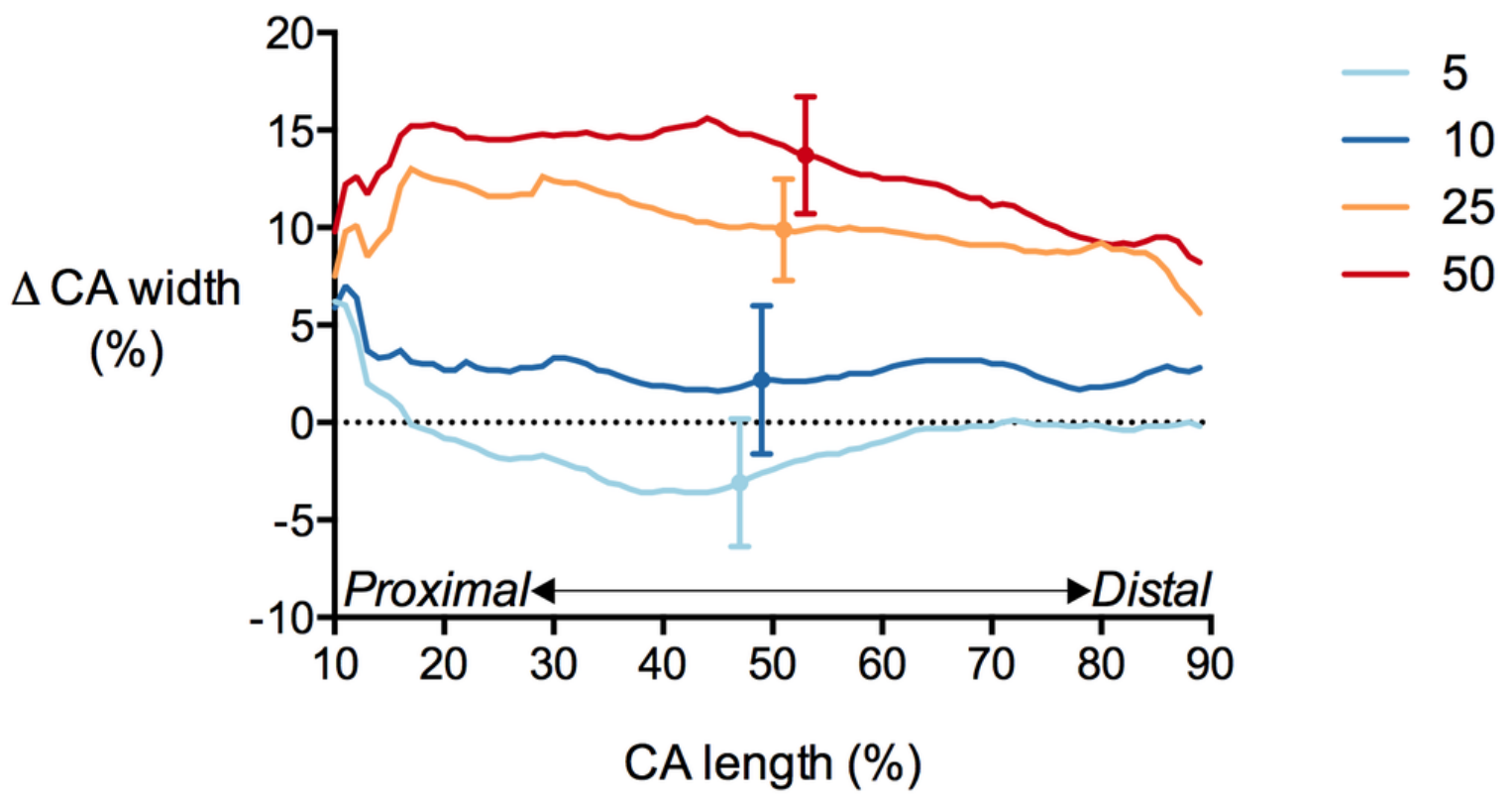

B

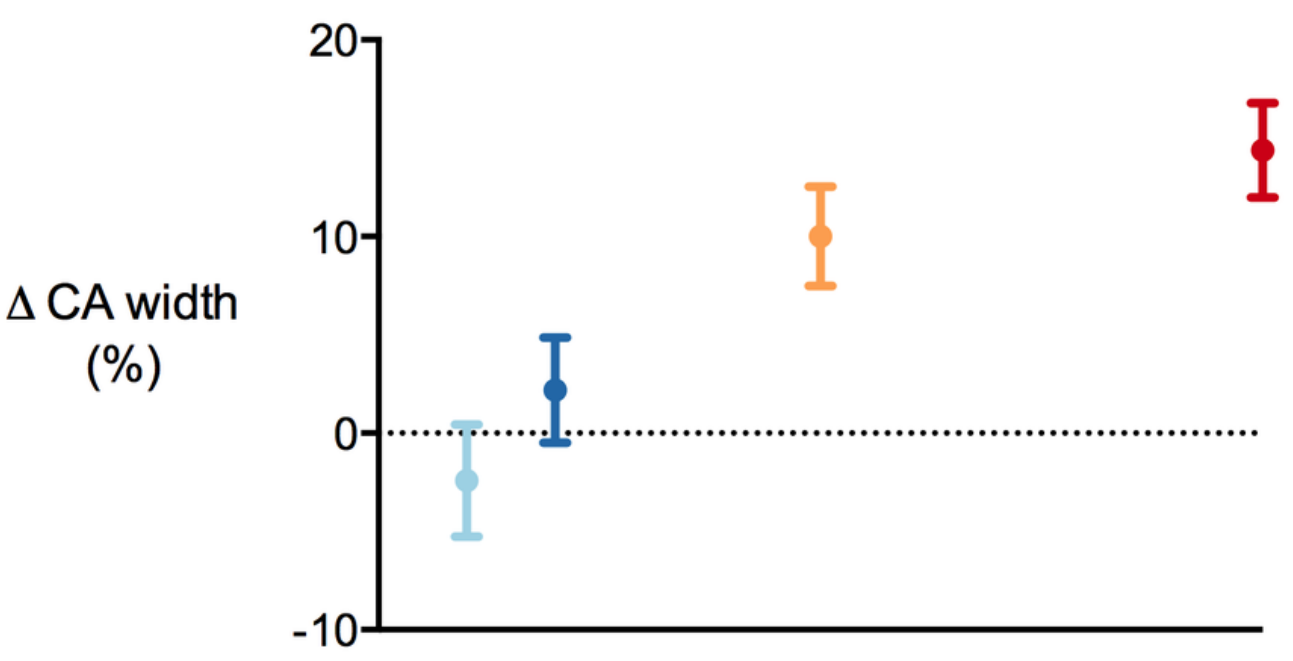

C

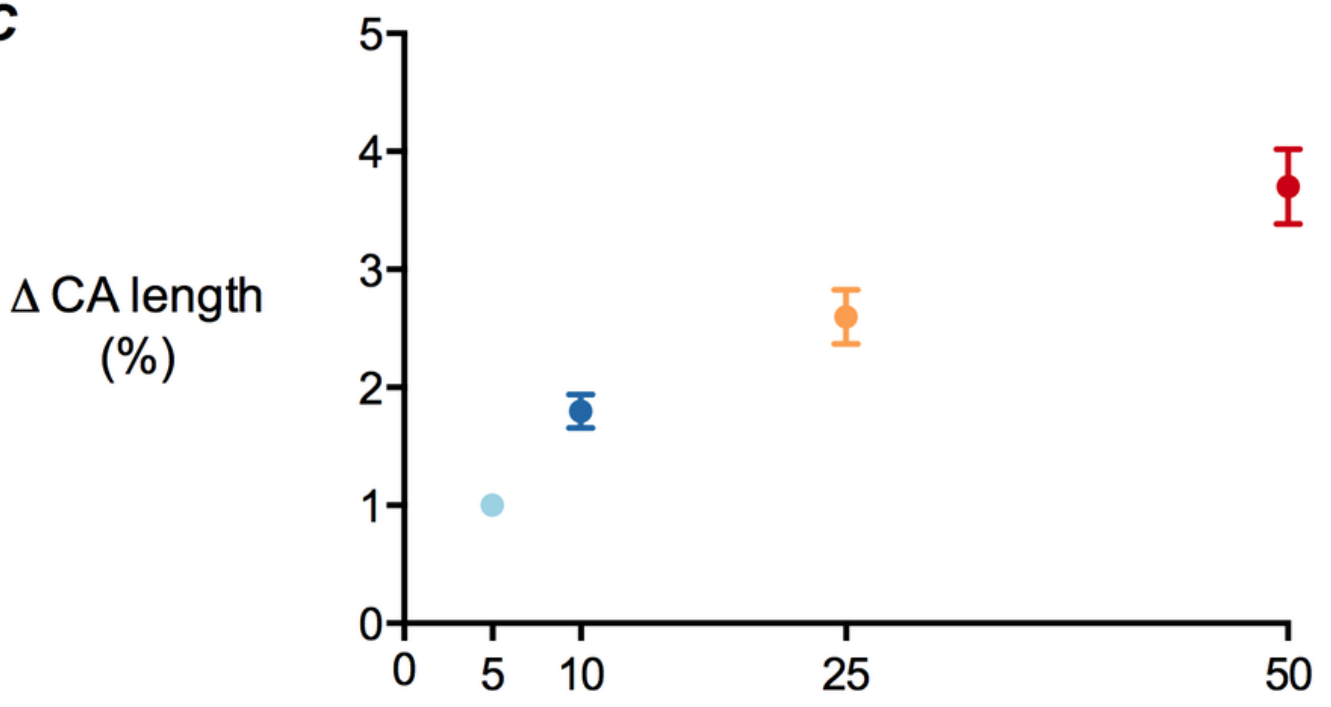




\section{Table 3(on next page)}

Tibialis anterior central aponeurosis width and length changes as a function of contraction intensity.

MVIC, Maximal Voluntary Isometric Contraction. 
1

\begin{tabular}{|c|c|c|c|c|c|}
\hline \multirow{2}{*}{ Central Aponeurosis Parameter } & \multicolumn{4}{|c|}{ Contraction Intensity (\% MVIC) } & \multirow{2}{*}{ P value } \\
\cline { 2 - 6 } & $\mathbf{5}$ & $\mathbf{1 0}$ & $\mathbf{2 5}$ & $\mathbf{5 0}$ & \\
\hline$\Delta$ Central aponeurosis width (mm) & $-0.8 \pm 3.8$ & $1.0 \pm 3.5$ & $4.4 \pm 3.6$ & $6.2 \pm 3.3$ & $<0.01$ \\
\hline$\Delta$ Central aponeurosis length (mm) & $1.6 \pm 0.5$ & $3.1 \pm 0.8$ & $4.3 \pm 1.1$ & $6.0 \pm 1.5$ & $<0.01$ \\
\hline
\end{tabular}

2 